\title{
A Simple and Rapid LC-MS/MS Method for Therapeutic Drug Monitoring of Lenalidomide
}

\author{
Neşet Neşetoğlu*, Cem Kaplan, Serap Sağlık Aslan and Durişehvar Özer Ünal \\ Istanbul University, Faculty of Pharmacy, Department of Analytical Chemistry. \\ İstanbul University, Drug Research Center, Beyazit, 34116, Istanbul, Turkey. \\ *Corresponding Author Email: neset.nesetoglu@istanbul.edu.tr \\ Received 01 October 2019, Revised 31 December 2019, Accepted 07 January 2020
}

\begin{abstract}
Immunomodulatory drugs lenalidomide (LENA) and pomalidomide (POMA) are synthetic compounds derived by modifying the chemical structure of thalidomide to improve its potency and reduce its side effects. LENA is used as a treatment for myeloma and blood disorders called myelodysplastic syndromes. The maximum clinical dose of LENA for some haematological cancers is generally $\leq 25 \mathrm{mg}$. Therapeutic drug monitoring (TDM) is important for the individualization of drug dosage. A highly sensitive and high performance liquid chromatography tandem mass spectrometry (HPLC-MS/MS) assay was developed and validated for the quantification of LENA in human plasma. LENA was extracted from human plasma by liquidliquid extraction by ethyl acetate and analysed using a reversed phase isocratic elution on a Poroshell 120 EC-C18, $(4.6-50 \mathrm{~mm}, 2.7 \mu \mathrm{m})$ column. A. $0.1 \%$ formic acid: methanol $(10: 90 \%$ $\mathrm{v} / \mathrm{v}$ ), was used as mobile phase and detection was performed by triple quadrupole mass spectrometry LC-MS/MS using jet stream electrospray ionization in negative mode. POMA was used as the internal standard (IS). Analyte and IS were detected by tandem mass spectrometry using multiple reaction monitoring (MRM) of precursor-product ion transitions with $0.100 \mathrm{~s}$ dwell time, at m/z $258.0>213.0$ for LENA and m/z $272.0>161.0$ for POMA. The calibration curves were consistently accurate and precise over the concentration range of 20 to $1000 \mathrm{ng} / \mathrm{mL}$ in plasma for LENA. This novel LC-MS/MS method competes with all the regulatory requirements and shows satisfactory accuracy and precision. It is sufficiently sensitive for the performance of pharmacokinetic, bioequivalence and TDM studies in humans.
\end{abstract}

Keywords: Lenalidomide, LC-MS/MS, Therapeutic drug monitoring.

\section{Introduction}

B-cell malignancies of the plasma cell cause multiple myeloma which is the second most common haematological malignancy. LENA (Fig. 1) is one of the novel compounds which is used for the treatment of this disease, it is based on the molecular structure of thalidomide and has an improved immunomodulatory effect and safety profile [1]. Thalidomide is a known human teratogen that causes severe life-threatening human birth defects. Use of LENA during pregnancy may cause anomaly or embryo-fetal death. LENA (3-(4-amino-1,3-dihydro-1-oxo-2Hisoindol-2-yl)-2,6-piperidinedione is an oral immunomodulatory drug (IMiD), with antineoplastic, antiangiogenic and antiinflammatory properties [2]. It has been used for the treatment of transfusion-dependent anemia due to lower intermediate-risk myelodysplastic syndromes (MDS) associated with a deletion $5 \mathrm{q}$ cytogenetic abnormality with or without additional cytogenetic abnormalities and in combination with dexamethasone for the treatment of multiple myeloma (MM) patients who have received at least one prior therapy [3]. LENA has a short halflife (3-4 h) and does not accumulate in plasma upon repeated dosing. Its pharmacokinetics are 
consistent across patient populations, regardless of the type of hematologic malignancy [4]. Studies have shown that LENA may work through various mechanisms in different hematologic malignancies. These mechanism involved direct cytotoxicity as well as through indirect effects on tumor immunity. Thus the differential efficacy noted with LENA therapy among various disease states can possibly be explained individual's immune status and disease specific pathophysiology [5].

Thalidomide (a)

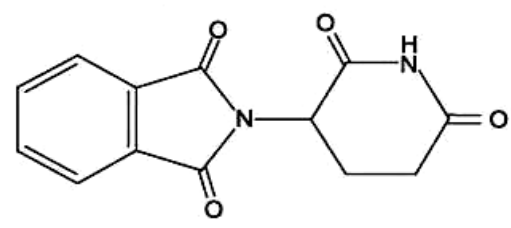

Lenalidomide (b)

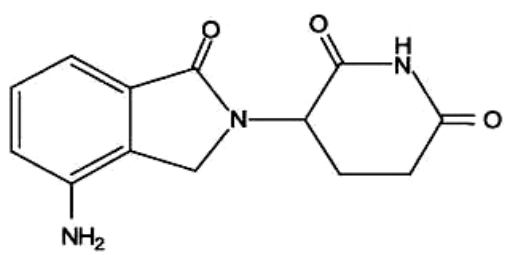

Pomalidomide (c)

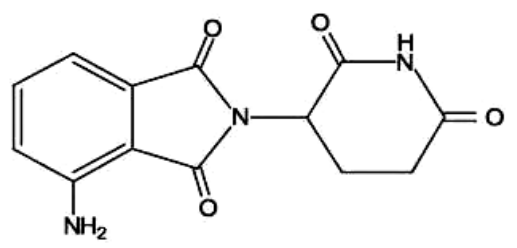

Figure 1. Chemical structure of Thalidomide (a), Lenalidomide (b), Pomalidomide (c)

TDM is the quantification and interpretation of drug concentrations in blood to optimize pharmacotherapy. It considers the interindividual variability of pharmacokinetics and thus enables personalized pharmacotherapy. In psychiatry and neurology, patient populations that may particularly benefit from TDM are children and adolescents, pregnant women, elderly patients, individuals with intellectual disabilities, patients with substance abuse disorders, forensic psychiatric patients or patients with known or suspected pharmacokinetic abnormalities [6]. TDM can be defined as the measurement of drug or active metabolite levels in biological samples (usually plasma) to individualise treatment by adapting drug dose and/or schedule to ultimately improve efficacy and/or reduce toxicity. Although many measures have been developed to reduce the side effects of chemotherapy, they remain a common problem and in some cases may even be life-threatening [7]. Immunoassays are not validated for alternative matrices that may have clinical utility for special populations, and they may not provide analytical measurement ranges sufficient to support selected sparing protocols or pharmacokinetic studies. Several immunoassays produce results that have a positive bias compared to chromatographic methods, due to metabolite cross-reactivity of the detection antibodies [8]. Application of LC-MS/MS to TDM has improved specificity of owing to the several opportunities to separate the components of the matrix and separate drugs and drug metabolites from one another.

The aim of TDM of LENA is to individualize therapeutic regimens for maximizing patient benefit. It is unnecessary to employ TDM for the majority of medications, and it is used mainly for monitoring drugs with narrow therapeutic ranges, drugs with marked pharmacokinetic variability, medications for which target concentrations are difficult to monitor, and drugs known to cause therapeutic and adverse effects [9]. The results from this trial demonstrated that daily doses of LENA equal to or greater than $75 \mathrm{mg}$ can result in significant myelo suppression. Although throm-bocytopenia was also observed, it was the severity of neutro-paenia induced by the treatment that proved dose limiting [10]. The maximum approved therapeutic dose of LENA is $25 \mathrm{mg}$ daily for patients with RRMM (relapsed and refractory multiple myeloma) and $10 \mathrm{mg}$ daily for patients with MDS. The maximum clinical dose of LENA for other haematological cancers is generally $\leq 25 \mathrm{mg}$. In terms of pharmacokinetic disposition, LENA undergoes minimum metabolism, and $82 \%$ of the LENA dose is eliminated unchanged via urinary excretion. Renal impairment reduces the elimination of LENA, thereby increasing drug concentration in plasma. As patients with MM or MDS often have compromised renal function due to their age or comorbidities, plasma LENA exposure is generally higher in these patients than in young 
healthy volunteers. Dose adjustment according to renal function has been recommended for patients with moderate or worse renal impairment. Gastrointestinal toxicity is extremely common during lenalidomide therapy, and occurs as nausea, vomiting, and diarrhea or constipation, but is usually mild to moderate in severity [11]. The most frequently reported adverse events were related to blood and lymphatic system disorders, skin and subcutaneous tissue disorders, gastrointestinal disorders.

Several chromatographic methods including spectrophotometric [12] and fluorimetric [13] methods were developed for the determination of LENA in pharmaceutical formulations such as bulk materials and capsules. Lopez et. al developed a method with using HPLC-UV chromatographic system and the analysis results of LENA calibration curve concentrations were in the range of 100 to 950 $\mathrm{ng} / \mathrm{mL}$ [14]. Furthermore there are also assays with using LC-MS/MS technique. Hasnain et al. worked with Box-Behnken experimental design [2]. Liu et al. developed a method to simultaneously quantify LENA and flavopiridol in plasma [15]. Mass spectrometry provides an alternative assay approach, relying on the discriminating power of mass analyzers to select a specific analyte and ion current measurements for quantitation. In the field of analytical chemistry, many small molecule analytes (e.g. drug metabolites, hormones, protein degradation products, and pesticides) are routinely measured using this approach at high throughput with great precision $(\mathrm{CV}<5 \%)$.

Electrospray ionization (ESI) followed by two stages of mass selection: first quadrupole mass filter (MS1) allows only ions of the target mass pass through (precursor, parent ion). In the collision cell precursor ions strikes by collision gas molecules generating product ions and neutral fragments. The second quadrupole (MS2) serves as a mass filter for the product ions produced in the collision cell for quantitate analysis of a target compound. As a consequence collectively generating a selected reaction monitoring (plural MRM) assay [16].
Among the currently available bioanalytical techniques LC-MS/MS has gained a considerable attention in recent years and has been emerged as the preeminent analytical tool for pharmaceutical and biomedical analysis because of its high speed, low time consumption, better resolution and better sensitivity.

The present study describes the development and validation of an HPLC method coupled with HPLC-MS/MS for the determination of LENA in human plasma for TDM study. The proposed method is simple, highly sensitive and it has high-throughput and reproducible.

\section{Materials and Methods \\ Chemicals and reagents}

LENA working standard was certified to contain $100.30 \%$ purity for LENA. Internal standard was certified to contain $99.70 \%$ purity for POMA. LC-MS Hyper grade methanol was used as an organic solvent and obtained from Merck (Darmstadt, Germany). Standard preparation and further dilution was made by methanol. The HPLC grade Merck (Darmstadt, Germany) ethyl acetate was used in liquid-liquid extraction. Ultrapure water was obtained from a Milli-Q water system (Millipore, Barnstead). HPLC grade formic acid used in mobile phase preparation was supplied from Merck (Darmstadt, Germany).

\section{Chromatographic conditions}

Agilent $1260 \quad$ Infinity Liquid Chromatography system and Triple Quadrupole QQQ-6460 dedector were used in this study. Analytical separation was carried out on a Poroshell 120 EC-C18, (4.6 - $50 \mathrm{~mm}, 2.7 \mu \mathrm{m})$ chromatographic column and maintained at $30^{\circ} \mathrm{C}$. The LC mobile phase was consisted of methanol: $0.1 \%$ formic Acid (90:10\%, v/v). The flow rate of the analytical method was $0.8 \mathrm{~mL} / \mathrm{min}$. The injection volume was $3.0 \mu \mathrm{L}$ and the analysis runtime was $2.0 \mathrm{~min}$.

\section{Mass spectrometry conditions}

Detection was carried out Agilent 6460 triple quadrupole MS/MS combining with Agilent Jet Stream Electrospray ionization (AJS-ESI) 
operated in the negative ion mode. Quantification was performed using multiple reaction monitoring (MRM) of the transitions of $\mathrm{m} / \mathrm{z} 258.0>213.0$ for LENA and m/z $272.0>161.0$ for POMA, with the dwell time of $100 \mathrm{~ms}$. Ultrapure (99.999\%) nitrogen gas was used in the collision cell for generating product ions and fragments. The optimized conditions were as follows; nebulizer pressure, drying gas temperature, sheath gas temperature, drying gas flow, sheath gas flow, capillary, and nozzle voltage. The MRM transitions and the related optimized fragmentor voltage were $140 \mathrm{~V}$ for LENA and $120 \mathrm{~V}$ for POMA. Collision energy were $11 \mathrm{~V}$ for analyte and $14 \mathrm{~V}$ for the IS.

\section{Preparation of solutions}

Standard stock solutions of LENA 1.0 $\mathrm{mg} / \mathrm{mL}(\mathrm{w} / \mathrm{v}$ ) and POMA (IS) $1.0 \mathrm{mg} / \mathrm{mL}(\mathrm{w} / \mathrm{v})$ were separately prepared in $5 \mathrm{~mL}$ volumetric flasks with methanol. Working solutions for calibration and quality control samples were prepared from the stock solution by diluting with methanol. Spiked plasma calibration standard solutions and quality control sample solutions were prepared at concentrations of $20,50,100$, 200, 500, $1000 \mathrm{ng} / \mathrm{mL}$ and 30, 250, 750 $\mathrm{ng} / \mathrm{mL}$, respectively. IS working solution $(50.0$ $\mu \mathrm{g} / \mathrm{mL}$ ) was prepared by diluting by the stock solution.

\section{Plasma sample preparation}

A simple and rapid extraction procedure was developed which provides a consistent matrix composition between samples and minimizes the potential for concentration dependent variation in protein binding. $500 \mu \mathrm{L}$ of plasma samples were taken into a polypropylene tubes and $100 \mu \mathrm{L}$ of IS added. Sample preparation involved a liquid-liquid extraction with ethyl acetate. $1.0 \mathrm{~mL}$ of ethyl acetate was added to each tube and vortexed for $2.5 \mathrm{~min}$ at $2800 \mathrm{rpm}$. Then samples were centrifuged at $5000 \mathrm{rpm}$ for 10 $\min$ at $20^{\circ} \mathrm{C}, 0.75 \mathrm{~mL}$ of supernatant layer was transferred to the vial and analyzed immediately.

\section{Method validation} Selectivity

The analytical method should be able to differentiate the analyte(s) of interest and IS from endogenous components in the matrix or other components in the sample. Selectivity should be proved using at least 6 individual sources of the appropriate blank matrix, which are individually analysed and evaluated for interference.

The selectivity of the method towards endogenous plasma matrix components and component medications was assessed in human blank plasma.

\section{Linearity}

Linearity demonstrates the accurate measurement of concentrations of spiked samples. For this purpose, plasma samples spiked at 6 concentration levels (20-50-100-200-500-1000 $\mathrm{ng} / \mathrm{mL}$ ) were extracted. For the quantification of the samples a calibration curve was constructed by spiking blank plasma at 6 concentration levels (20$1000 \mathrm{ng} / \mathrm{mL}$ ).

Calibration curves were constructed using matrix matched calibration standard solutions by plotting the peak area of the quantitative ion of each analyte versus concentrations. The determination coefficients $\left(\mathrm{r}^{2}\right)$ were consistently greater than 0.999 during the course of validation.

\section{Accuracy and precision}

Accuracy was assessed on samples spiked with known amounts of the analyte, the quality control samples (QC samples). The QC samples were spiked independently from the calibration standards, using separately prepared stock solutions. Accuracy was determined by analysing 6 samples per level at a minimum of 3 concentration levels which are covering the calibration curve range.

Precision was demonstrated for the LOQ, low, medium and high QC samples, within a single run and between different runs, i.e. using the same runs and data as for the demonstration of 
accuracy. Precision is expressed as \%CV (coefficient of variation) for 18 determinations. Intra assay precision and accuracy of LENA were calculated at $30 \mathrm{ng} / \mathrm{mL}, 250 \mathrm{ng} / \mathrm{mL}$ and 750 $\mathrm{ng} / \mathrm{mL}$ levels for the six replicates, each of the same analytical run. Inter assay precision and accuracy was calculated after the replicates in three different analytical runs.

\section{Matrix effect}

Liquid chromatography coupled to mass spectrometry (LC-MS) is a powerful tool for bioanalysis. Reliable bioanalysis requires the characterization of the matrix effect, i.e. influence of the endogenous or exogenous compounds on the analyte signal intensity.

Matrix effect is evaluated using pool plasma from different sources to prepare samples. For analyte and the IS, the matrix factor (MF) are calculated in each matrix, by calculating the ratio of the peak area in the presence of matrix (measured by analysing blank matrix spiked with analyte at a concentration of quality conrol samples after extraction), to the peak area in absence of matrix (pure solution of the analyte). The IS normalised MF is calculated by dividing the MF of the analyte by the MF of the IS.

Extracted and aqueous samples were compared to determine the MF for analyte and IS. IS normalized MF for individual lot is also determined. The calculated MF of all LQC (low quantity concentration) and HQC (high quantity concentration) samples must be within $85-115 \%$ of their nominal concentration.

\section{System suitability}

System suitability tests are an integral part of GC and LC methods. These tests are used to verify that the chromatographic system is adequate for the intended analysis. The tests are based on the concept that the equipment, electronics, analytical operations, and samples analyzed constitute an integral system that can be evaluated as such. RS (resolution) is a function of the number of theoretical plates, $\mathrm{N}$ (number of theoretical plates) (also referred to as efficiency) and k' (capacity factor).

\section{Results and Discussion}

The analysis was performed in the mass spectrometer using an electrospray (ESI) in the negative ionization mode. The reliability of the method was assessed on the basis of linearity, sensitivity, selectivity, precision and accuracy. On the basis of the results obtained for the above parameters, the combination of mobile phase, flow rate and dedector parameters were selected for validation. Out of several tried combinations, results suggested a combination of $0.1 \%$ formic acid: methanol (10:90\% v/v) ratio of mobile phase, flow rate $0.800 \mathrm{~mL} / \mathrm{min}$ which resulted in a retention time of $0.568 \mathrm{~min}$ for LENA and 0.543 min for the IS. LC-MS/MS operation parameters were carefully optimized for the determination of LENA. Both analyte and IS were detected by tandem mass spectrometry using MRM of precursor-product ion transitions with $0.100 \mathrm{~s}$ dwell time at $\mathrm{m} / \mathrm{z} 258.0>213.0$ for LENA and $\mathrm{m} / \mathrm{z} 272.0>161.0$ for POMA. The mass spectrometer was tuned initially in both positive and negative ionization modes for LENA. It was observed that the signal intensity of negative ion was much higher than that of positive ion. Parameters, such as capillary and nozzle voltage, desolvation temperature, ESI source temperature and flow rate of desolvation gas and cone gas, were optimized to obtain the optimum intensity of molecules of LENA and IS for quantification.

The optimized mass analyzer conditions were as follows; nebulizer pressure 45 psi, drying gas temperature $320^{\circ} \mathrm{C}$, sheath gas temperature $250^{\circ} \mathrm{C}$, drying gas flow $5(\mathrm{~L} / \mathrm{min})$, sheath gas flow $11(\mathrm{~L} / \mathrm{min})$, capillary $3500 \mathrm{~V}$ and nozzle voltage $500 \mathrm{~V}$. The MRM transitions and the related optimized fragmentor voltage were $140 \mathrm{~V}$ for LENA and $120 \mathrm{~V}$ for POMA. Collision energy were $11 \mathrm{~V}$ for analyte and $14 \mathrm{~V}$ for the IS.

MRM is a highly specific and sensitive mass spectrometry technique that can selectively quantify compounds within complex mixtures. This technique uses a triple quadrupole MS that firstly targets the ion corresponding to the 
compound of interest with subsequent fragmentation of that target ion to produce a range of product ions. One (or more of these fragment product ions can be selected for quantification purposes. Only compounds that meet both these criteria, i.e. specific precursor ion and specific product ions corresponding to the mass of the molecule of interest are isolated within the mass spectrometer. Mass spectromerty MRM mode also provides us to analyze compounds with the same retention time (See Fig. 2 and Fig. 3).

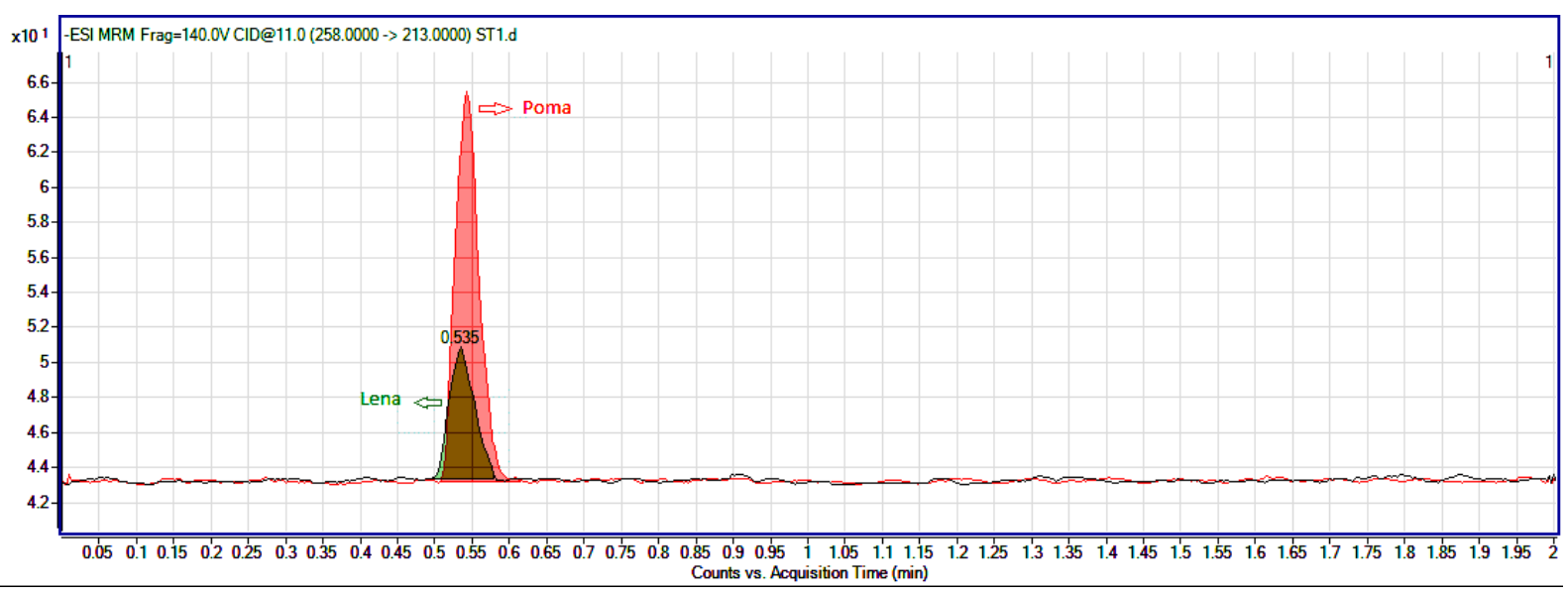

Figure 2. Overlaid chromatograms of lenalidomide and pomalidomide (IS) with using MRM mode

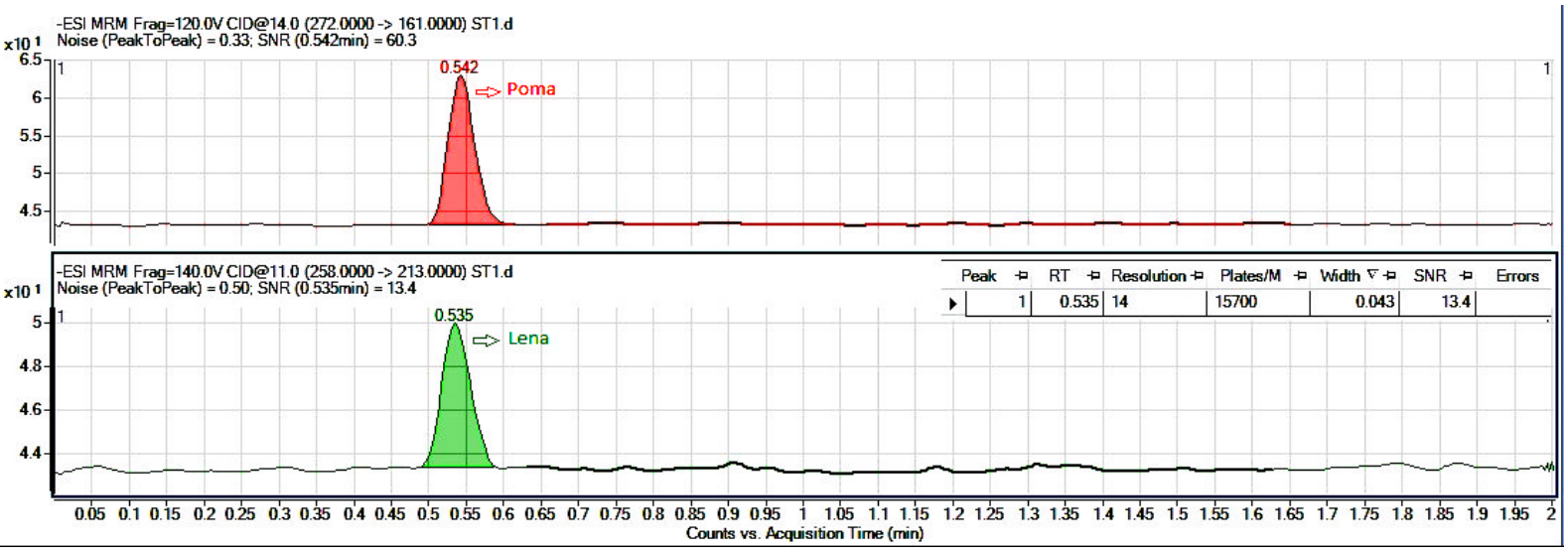

Figure 3. Extracted chromatograms of lenalidomide and pomalidomide (IS) with using MRM mode

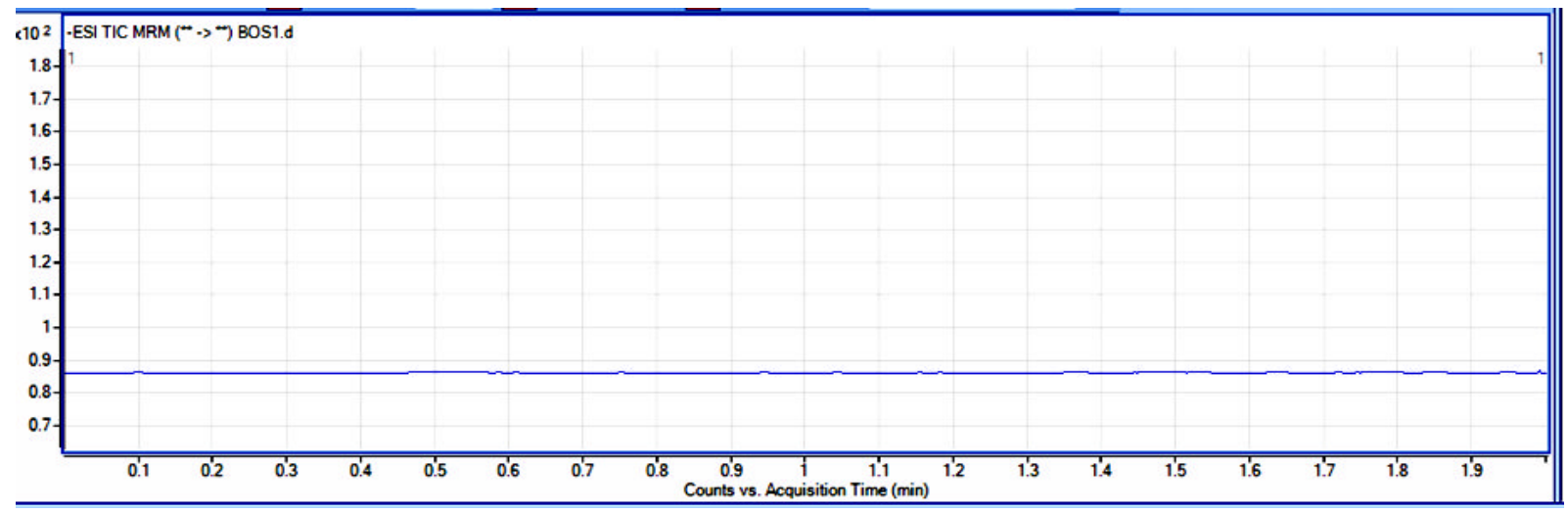

Figure 4. Chromatogram of blank plasma 
The selectivity was carried out using six different individual sources and analyzed using the proposed extraction method. The plasma batch did not show any interferences at the retention times of LENA and POMA. Representative chromatogram of blank plasma is given in Fig. 4 .

The calibration curve regression $\left(\mathrm{r}^{2}\right)$ for lenalidomide was found to be 0.99976 . Coefficient of determination $\left(\mathrm{r}^{2}\right)$ for validation was greater than 0.99 which provide the requirements of bioanalytical method validation guideline. Calibration curve's equation is $\mathrm{y}=0,001 \mathrm{x}$ 0,0173. Chen et. al. investigated plasma pharmacokinetic parameters of LENA in volunteers. The Cmax was found $390 \mathrm{ng} / \mathrm{mL}$ for young adults and $568 \mathrm{ng} / \mathrm{mL}$ for older adults in the clinical trial [4]. For the propose of Cmax value must be in the range of calibration curve. Linear concentration range of LENA was found to be accurate and precise from 20 to $1000 \mathrm{ng} / \mathrm{mL}$. LOD and LOQ values were determined as $4.8 \mathrm{ng} / \mathrm{mL}$ and $14.5 \mathrm{ng} / \mathrm{mL}$, respectively.

Two ultra-fast HPLC-MS/MS methods have been developed for the determination of LENA in plasma in the literature. While our linear range values are similar, there are differences in LOQ values due to ultra-fast HPLC and LC methods $[2,17]$.

Accuracy and precision of LENA were calculated at $30 \mathrm{ng} / \mathrm{mL}, 250 \mathrm{ng} / \mathrm{mL}$ and 750 $\mathrm{ng} / \mathrm{mL}$ levels for the six replicates. Coefficients of variation results were $4.60 \%, 3.75 \%$ and $1.65 \%$, respectively whereas accuracies were $103.02 \pm 3.02,99.06 \pm 0.94$ and $101.01 \pm 1.01$, respectively in human plasma (Table 1). Values are within the acceptance limit of $\pm 15 \%$. The results indicate that the method has good precision and accuracy.

Table 1. Lenalidomide accuracy and precision in QC samples.

\begin{tabular}{ccc}
\hline Conc. $(\mathbf{n g} / \mathbf{m L})$ & $\begin{array}{c}\text { Accuracy } \\
(\mathbf{\%} \text { Error })\end{array}$ & $\begin{array}{c}\text { Precision } \\
(\mathbf{\%} \mathbf{C V})\end{array}$ \\
\hline 30 & $103.02 \pm 3,02$ & 4.60 \\
250 & $99.06 \pm 0,94$ & 3.75 \\
750 & $101.01 \pm 1,01$ & 1.65 \\
\hline
\end{tabular}

The calculated MF coefficients of variation was $11.67 \%$ within $85-115 \%$ of their nominal concentration. Determination of matrix effect by the slope of the calibration curve is given in Fig. 5. The method showed that no matrix effect was found for human plasma for TDM routine analysis.

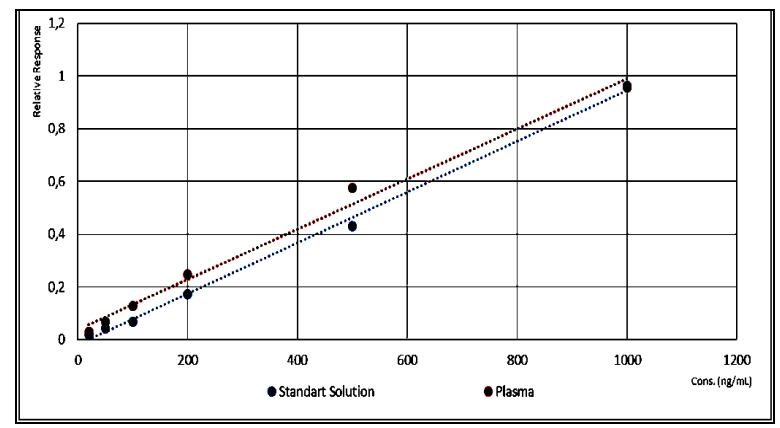

Figure 5. Determination of matrix effect by the slope of the calibration curve

System suitability parameters found as specified in guideline. Theorical plate number $(\mathrm{N})$ was calculated as 15700 , capacity factor (k') 9.8 , resolution (Rs) 14, tailing factor (T) 1.1 for LENA. Detailed parameters are given in Table 2.

Table 2. ICH guideline system suitability parameters, limits and results.

\begin{tabular}{lcc}
\hline Parameters & Limits & Results \\
\hline Capacity factor (k') & $>2$ & 9.80 \\
$\begin{array}{l}\text { Injection accuracy } \\
(\mathrm{RSD})\end{array}$ & $<\% 1, \mathrm{n} \geq 5$ & 0.49 \\
Resolution (RS) & $>2$ & 14 \\
Tailing factor (T) & $\leq 2$ & 1.1 \\
$\begin{array}{l}\text { Number of } \\
\text { theoretical plates }(\mathrm{N})\end{array}$ & $>2000$ & 15700 \\
\hline
\end{tabular}

For LENA, TDM aims to enhance drug efficacy, reduce toxicity or assist with diagnosis. Despite its apparent advantages, it has also limitations. Some large hospitals have services which provide support with drug monitoring and interpretation of results. Unless TDM is being used to forecast a dose or there are concerns about toxicity, samples should be taken at steady state (4-5 half-lives after starting therapy). TDM can optimise patient management and improve clinical outcomes [9]. 


\section{Conclusion}

A novel simple, economical, highthroughput and highly sensitive HPLC-MS/MS method was successfully developed and validated for the determination of LENA in human plasma. The method involved simple one step protein precipitation method for plasma sample preparation and short runtime $(2.0 \mathrm{~min})$ for analysis. The proposed method could be practical and reliable for pharmacokinetic and TDM study for LENA in human.

\section{References}

1. J. B. Bartlett, K. Dredge and A. G. Dalgleish, Nat. Rev. Cancer, 4 (2004) 314. doi: $10.1038 / \mathrm{nrc} 1323$.

2. M. S. Hasnain, S. Rao, M. K. Singh, N. Vig, A. Gupta, A. Ansari, P. Sen, P. Joshi and S. A. Ansari, Analyst, 138 (2013) 1581. doi: 10.1039/c2an36701g.

3. N. Chen, L. Wen, H. Lau, S. Surapaneni and G. Kumar, Cancer Chemo. Pharma., 69 (2012) 789.

doi: 10.1007/s00280-011-1760-3.

4. N. Chen, S. Zhou and M. Palmisano, Clin. Pharma., 56 (2017) 139. doi 10.1007/s40262-016-0432-1.

5. V. Kotla, S. Goel, S. Nischal1, C. Heuck, K. Vivek, B. Das and A. Verma, J. Hematol. Oncol., 2 (2009) 36. doi:10.1186/1756-8722-2-36

6. C. Hiemke, N. Bergemann, H. W. Clement, A. Conca, J. Deckert, K. Domschke, G. Eckermann, K. Egberts, M. Gerlach, C. Greiner, G. Gründer, E. Haen, U. Havemann-Reinecke, G. Hefner, R. Helmer, G. Janssen, E. Jaquenoud, G. Laux, T. Messer, R. Mössner, M. J. Müller, M. Paulzen, B. Pfuhlmann, P. Riederer, A. Saria, B. Schoppek, G. Schoretsanitis, M. Schwarz, M. Silva Gracia, B. Stegmann, W. Steimer, J. C. Stingl, M. Uhr, S. Ulrich, S. Unterecker, R. Waschgler, G. Zernig, G. Zurek and P. Baumann, Pharmacopsychiatry, 51 (2018) 9. doi: $10.1055 / \mathrm{s}-0043-116492$

7. C. Bardin, G. Veal, A. Paci, E. Chatelut, A. Astier, D. Leveque, N. Widmer and J. Beijnen, Eur. J. Cancer, 50 (2014) 2005. doi: 10.1016/j.ejca.2014.04.013

8. L. K. Johnson-Davis and G. A. McMillin, Chapter3 in Personalized Immunosuppression in Transplantation, Role of Biomarker Monitoring and Therapeutic Drug Monitoring, (2016) 57, M. Oellerich and A. Dasgupta (Eds).

doi:http://dx.doi.org/10.1016/B978-0-12800885-0.00003-5

9. R. A. Ghiculescu, Aust. Prescr., 31 (2008) 42.

doi: 10.18773/austprescr.2008.025.

$10 . \quad$ R. A. Sharma, W. P. Steward, C. A. Daines, R. D. Knight, K. J. O'Byrne, A. G. Dalgleish, Eur. J. Cancer., 42 (2006) 2318. doi:10.1016/j.ejca.2006.05.018.

11. A. P. G. Rodríguez, Adv. Ther., 28 (2011) 1. doi: $10.1007 / \mathrm{s} 12325-010-0104-8$

12. B. S. Sastry, S. Gananadhamu, S. V. S. G. B. Prasad and K. V. G. Raju, Int. J. Pharm. Tech. Res., 1 (2009) 416. http://sphinxsai.com/PTVOL3/PT=5\%20,G ANA\%20NADHAM\%20(416-419).pdf

13. I. A. Darwish, N. Y. Khalil, A. H. Bakheit and N. Z. Alzoman, Chem. Cent. J., 6 (2012) 118 . doi: 10.1186/1752-153X-6-118.

14. B. G. López, A. P. Pitarch, M. A. M. Gómez, B. P. Oltra, M. C. Martí and M. M. Sanjuán, J. Lab. Automation, 21 (2016) 806. doi: $10.1177 / 2211068216636570$

15. Q. Liu, K. L. Farley, A. J. Johnson, N. Muthusamy, C. C. Hofmeister, K. A. Blum, L. J. Schaaf, M. R. Grever, J. C. Byrd, J. T. Dalton and M. A. Phelps, Ther. Drug Monit., 30 (2008) 620.

doi:10.1097/FTD.0b013e318185813d.

16. L. Anderson and C. L. Hunter, Mol. Cell Proteomics, 5 (2006) 573. doi: 10.1074/mcp.M500331-MCP200

17. P. Ranganathan, V. Gunasekaran, I. Singhvi and M. J. Ansari, Saudi J. Biol. Sci., 26 (2019) 1843. doi: $10.1016 /$ j.sjbs.2018.02.006 